\title{
A Genomics-Based Approach Identifies a Thioviridamide-Like Compound with Selective Anticancer Activity
}

\author{
Luca Frattaruolo, ${ }^{\dagger,}$ Rodney Lacret, ${ }^{\dagger}$ Anna Rita Cappello, ${ }^{*}$ and Andrew W. Truman ${ }^{*}{ }^{\dagger}$ (i) \\ †Department of Molecular Microbiology, John Innes Centre, Colney Lane, Norwich, NR4 7UH, United Kingdom \\ *Department of Pharmacy, Health and Nutritional Sciences, University of Calabria, Rende, Italy
}

\section{Supporting Information}

ABSTRACT: Thioviridamide is a structurally novel ribosomally synthesized and post-translational modified peptide (RiPP) produced by Streptomyces olivoviridis NA005001. It is characterized by a structure that features a series of thioamide groups and possesses potent antiproliferative activity in cancer cell lines. Its unusual structure allied to its promise as an anticancer compound led us to investigate the diversity of thioviridamide-like pathways across sequenced bacterial genomes. We have isolated and characterized three diverse members of this family of natural products. This characterization is supported by transformation-associated recombination cloning and heterologous expression of one of these

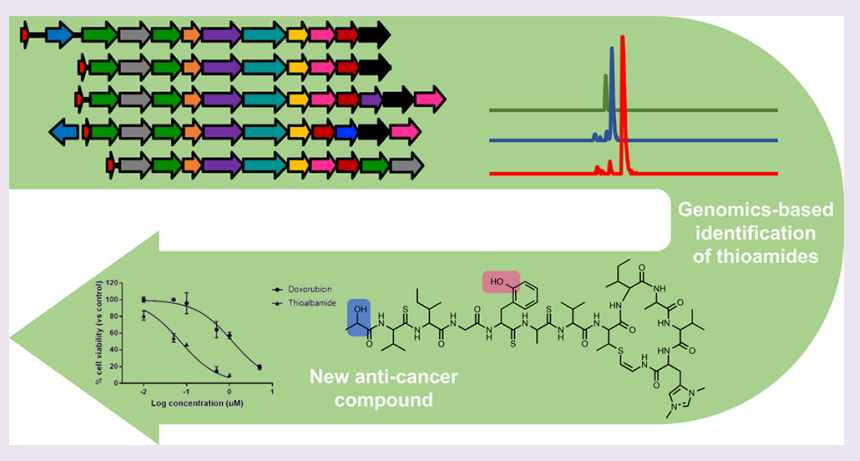
compounds, thiostreptamide S4. Our work provides an insight into the diversity of this rare class of compound and indicates that the unusual $N$-terminus of thioviridamide is not introduced biosynthetically but is instead introduced during acetone extraction. A detailed analysis of the biological activity of one of the newly discovered compounds, thioalbamide, indicates that it is highly cytotoxic to cancer cells, while exhibiting significantly less activity toward a noncancerous epithelial cell line.

$\mathrm{R}$ ibosomally synthesized and post-translationally modified peptides (RiPPs) are a class of natural products with high chemical diversity, which results from the wide array of maturation processes that the genetically encoded precursor peptide may undergo. ${ }^{1,2}$ This diversity is reflected in the wide variety of biological activities that these compounds possess. Due to their broad pharmacologic potential, this rapidly growing class of natural products is currently a focus of biotechnology and pharmaceutical research. ${ }^{3,4}$ Thioviridamide (1, Figure 1a) is a RiPP biosynthesized by Streptomyces olivoviridis NA005001 and is characterized by potent antiproliferative and pro-apoptotic activity in several cancer cell lines. ${ }^{5}$ This novel peptidic compound features an $\mathrm{N}$-terminal 2hydroxy-2-methyl-4-oxopentanoyl group, a $\beta$-hydroxy- $N_{1}, N_{3}$ dimethylhistidinium (hdmHis) residue, and a $S$-(2-aminovinyl)cysteine (AviCys) residue ${ }^{6}$ that forms part of a macrocycle. ${ }^{7} \mathrm{~A}$ very unusual feature of $\mathbf{1}$ is the presence of five thioamide groups $^{8}$ in place of backbone amide groups. Recently, the thioviridamide $(t v a)$ biosynthetic gene cluster (BGC) was identified and confirmed by heterologous expression. 9 This demonstrated the ribosomal origin of this molecule, which derives from a 13 amino acid core peptide at the $\mathrm{C}$ terminus of the TvaA precursor peptide (Figure 1b). An additional 11 proteins encoded by this gene cluster (TvaB-TvaL) are predicted to be involved in the maturation of the precursor peptide into $\mathbf{1}$, although little is known about individual steps in this pathway. The promising antitumor activity, unprecedented structure, and poorly understood biosynthesis of thioviridamide led us to investigate the extent and diversity of this new family of natural products.

Here, we report the identification and characterization of three new thioviridamide-like pathways. Chemical and biological characterization of these new thioviridamide-like molecules (TLMs) demonstrates the diversity of this type of RiPP, which is likely to be a subclass of a wider thioamide family of RiPPs. ${ }^{10}$ Our work indicates that the reported thioviridamide structure may be an unnatural derivative of the true pathway product. Gene inactivation and heterologous expression of a transformation-associated recombination (TAR) cloned gene cluster supported this characterization. Finally, we show that a TLM produced by Amycolatopsis alba DSM 44262 is highly cytotoxic toward various tumor cell lines and yet is 6-fold less active toward a noncancerous epithelial cell line, thus exhibiting medicinally promising selectivity toward cancerous cells.

\section{RESULTS AND DISCUSSION}

Genome Mining to Identify Thioviridamide-Like Pathways. A BLAST search using the $\mathrm{YcaO}$ domain protein $\mathrm{TvaH}$ yielded 22 proteins with over $50 \%$ identity. The genomic regions surrounding their respective genes were then analyzed by a MultiGeneBlast ${ }^{11}$ homology search using the tva BGC as

Received: August 7, 2017

Accepted: October 2, 2017

Published: October 2, 2017 


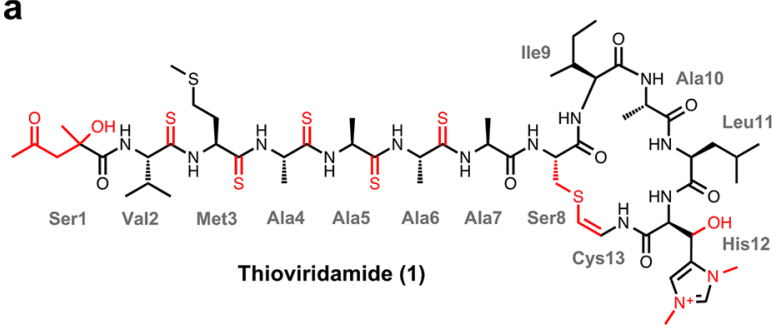

b

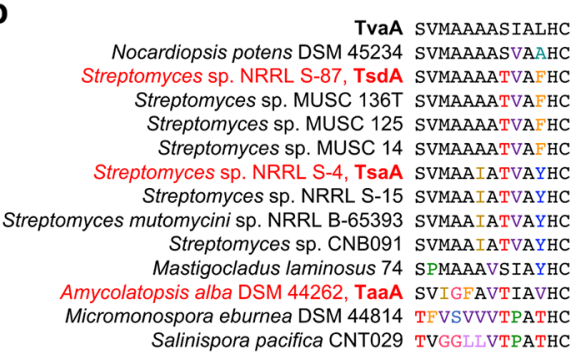

C

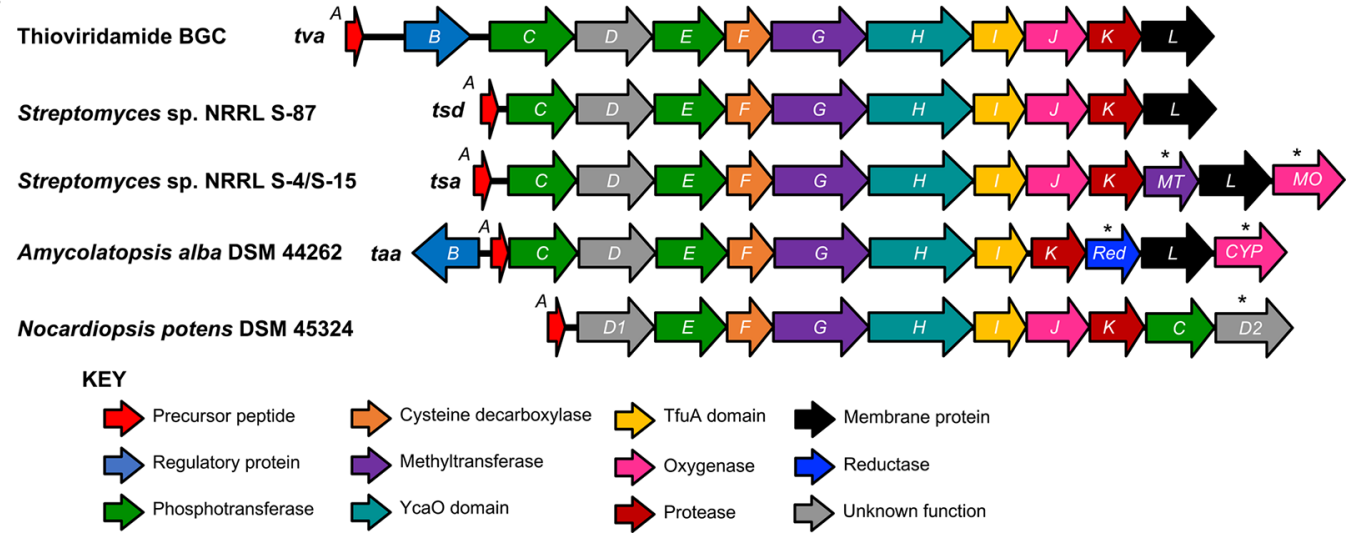

Figure 1. Identification of thioviridamide-like pathways. (a) Structure of thioviridamide with each post-translational modification highlighted red. (b) Alignment of the core peptides from every sequenced thioviridamide-like BGC. All residues that differ from TvaA are colored. (c) Comparison of the gene clusters investigated in this study $(\mathrm{MT}=$ methyltransferase; $\mathrm{MO}=$ flavin-dependent oxygenase; Red $=$ reductase; $\mathrm{CYP}=\mathrm{P} 450 ; *=$ putative new biosynthetic enzymes). A comparison of all 14 BGCs is provided in Figure S1.

the query, which identified 14 closely related TLM-like BGCs in bacterial genomes. All were in Actinobacteria, with the intriguing exception of the cyanobacterium Mastigocladus laminosus. These all encode putative precursor peptides (Figure 1b) with high sequence homology to TvaA and feature a partially conserved set of tailoring genes (Figure $1 \mathrm{c}$ and Figure S1). We selected five publicly available strains that we predicted would collectively provide much of the diversity in this family of compounds: A. alba DSM 44262, Streptomyces sp. NRRL S-4, Streptomyces sp. NRRL S-15, Streptomyces sp. NRRL S-87, and Nocardiopsis potens DSM 45234. These gene clusters have homologues of most of the genes involved in thioviridamide maturation (from $\mathrm{TvaC}$ to TvaL), except for the A. alba cluster, which lacks a hydroxylase-encoding tvaj-like gene (Figure 1c). In addition, only the $A$. alba BGC encodes a putative homologue of $\mathrm{TvaB}$, a regulatory protein encoded in the tva BGC. Intriguingly, the gene clusters in Streptomyces sp. NRRL S-4/S-15 and A. alba DSM 44262 contain additional genes that could be involved in further post-translational modification steps (Figure 1c).

Production of Thioviridamide-Like Molecules. The putative core peptide sequences and associated set of tailoring enzymes allowed us to predict candidate masses for each TLM. Therefore, each strain was fermented in multiple culture conditions and screened by liquid chromatography-mass spectrometry (LC-MS) for TLM production. While the production medium reported ${ }^{5}$ for $\mathbf{1}$ provided no candidate molecules, a solid version of bottromycin production medium ${ }^{12}$ (BPM) yielded compounds with masses compatible with TLMs derived from the respective precursor peptides (Figure 2a), with the exception of $N$. potens DSM 45234. A. alba DSM 44262 produced a compound (thioalbamide, 2 , Figure $2 \mathrm{~b}$ ) with $\mathrm{m} / z 1329.6349$ (Figure 2a), corresponding to the molecular formula $\mathrm{C}_{61} \mathrm{H}_{97} \mathrm{~N}_{14} \mathrm{O}_{11} \mathrm{~S}_{4}{ }^{+}$(calculated $\mathrm{M}^{+}$: 1329.6339). High- resolution (HR) $\mathrm{MS}^{2}$ analysis of this molecule provided a fragmentation pattern that supported a thioviridamide-like structure, including the presence of thioamides (and associated losses of $\mathrm{H}_{2} \mathrm{~S},-33.99 \mathrm{Da}$ ), fragmentation consistent with the predicted linear portion of the molecule, and an $\mathrm{MS}^{2}$ fragment $(m / z$ 607.3376, Figure 2 and Figure S2) that correlated with a (2-aminovinyl)-3-methyl-cysteine (AviMeCys) containing macrocycle (predicted $\mathrm{m} / z$ 607.3385).

The gene clusters for Streptomyces sp. NRRL S-4 and Streptomyces sp. NRRL S-15 are effectively identical, and each strain produced a compound (thiostreptamide S4, 3, Figure $2 b$ ) with $\mathrm{m} / z 1377.55$ that eluted at an identical retention time (Figure S3), corresponding to the molecular formula $\mathrm{C}_{60} \mathrm{H}_{93} \mathrm{~N}_{14} \mathrm{O}_{11} \mathrm{~S}_{6}^{+}$(Streptomyces sp. NRRL S-4 product $\mathrm{m} / \mathrm{z}$ 1377.5479; calculated $\left.\mathrm{M}^{+}=1377.5467\right)$. As with 2 , HRMS $^{2}$ analysis of this molecule provided a fragmentation pattern that supported a thioviridamide-like structure, including multiple thioamides and a putative AviMeCys-containing macrocycle fragment (obsd. $m / z$ 687.3260, pred. $m / z$ 687.3283, Figure $2 \mathrm{~b}$ and Figure S4). This preliminary analysis indicated that the S-4 and S-15 pathways produce identical compounds, although differences in stereochemistry cannot be ruled out. Streptomyces sp. NRRL S-87 produced a compound (thiostreptamide S87, 4, Figure $2 \mathrm{~b}$ ) with $\mathrm{m} / \mathrm{z} 1305.4871$, corresponding to the molecular formula $\mathrm{C}_{56} \mathrm{H}_{85} \mathrm{~N}_{14} \mathrm{O}_{10} \mathrm{~S}_{6}{ }^{+}$(calculated $\mathrm{M}^{+}$: 1305.4892). As before, HRMS ${ }^{2}$ analysis provided thioviridamide-like fragments that were consistent with the predicted precursor peptide (Figure $2 \mathrm{~b}$ and Figure S5).

The most striking structural feature of thioviridamide is a contiguous sequence of five thioamide-containing residues, which subtly differs from the TLMs that we isolated. Instead, exact mass and $\mathrm{MS}^{2}$ data signified three noncontiguous thioamide linkages in 2, and four in $\mathbf{3}$ and $\mathbf{4}$ (Figure 2b). UV absorption spectra of each compound provided maxima of 
a

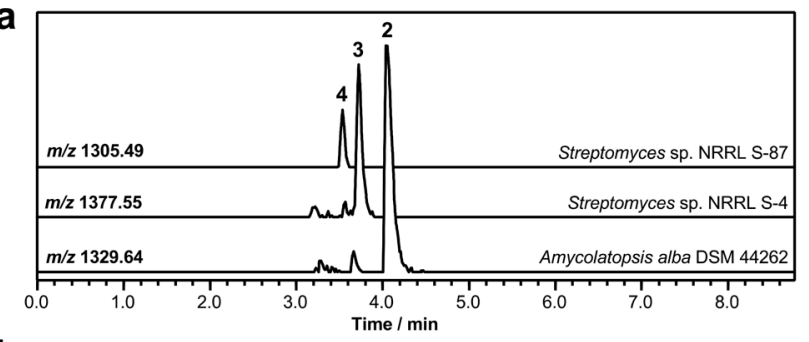

b

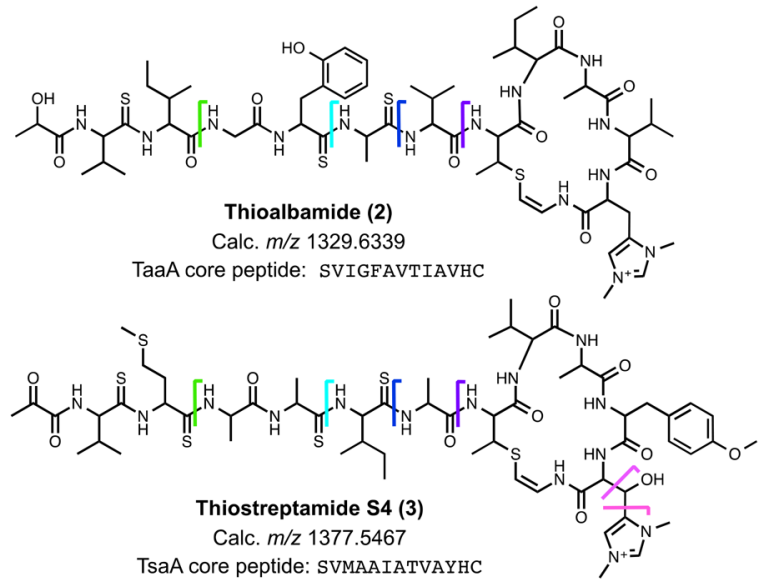

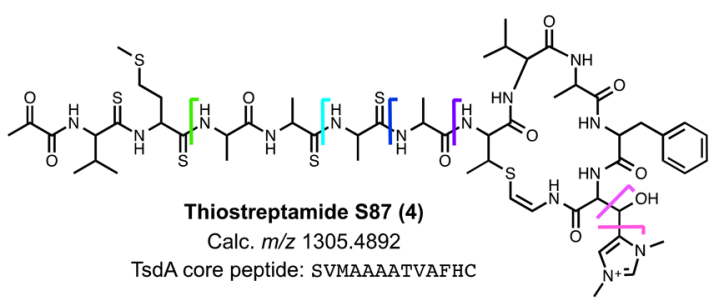
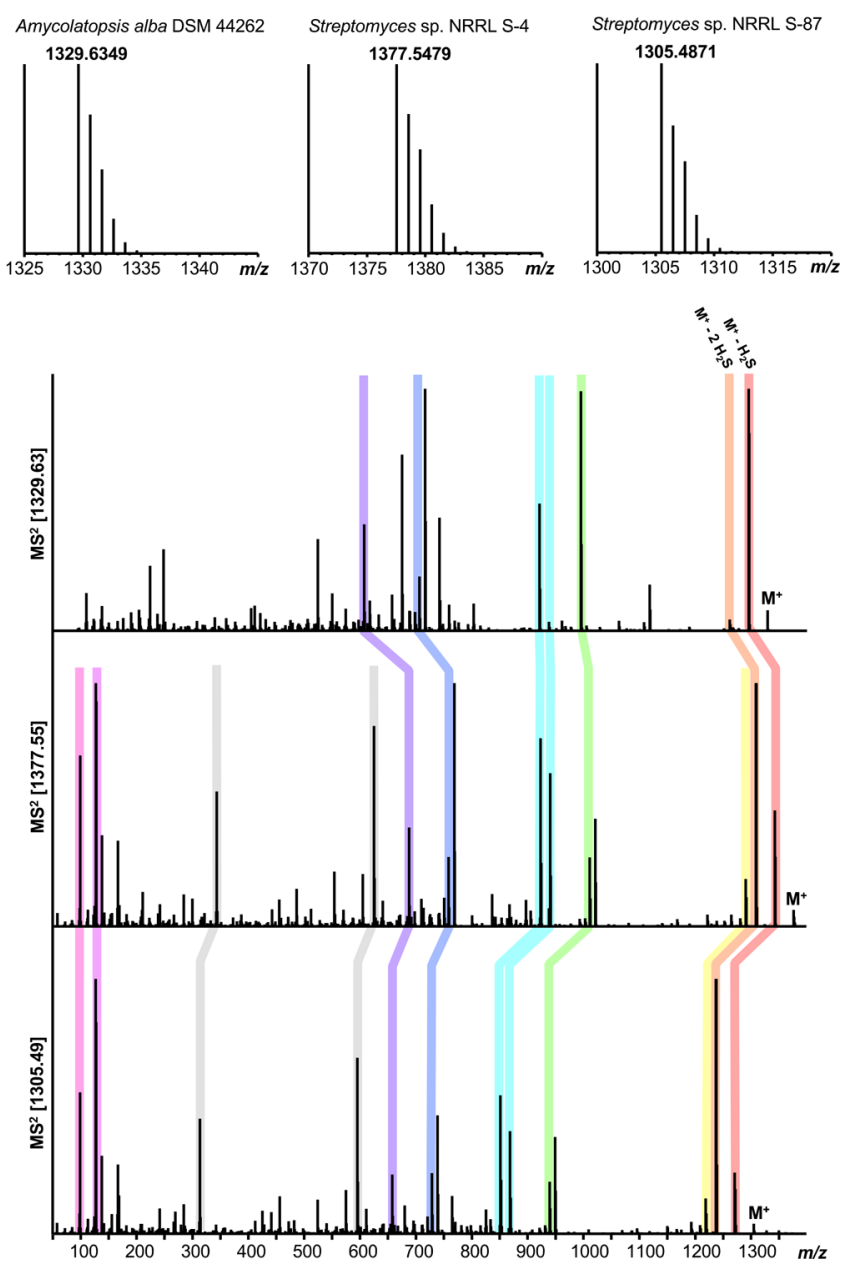

Figure 2. Identification of novel TLMs. (a) Extracted ion chromatograms from A. alba DSM 44262, Streptomyces sp. NRRL S-4, and Streptomyces sp. NRRL S-87 showing relative levels of production and exact masses for each compound. (b) Structures of each compound alongside MS ${ }^{2}$ data indicating analogous fragments from each compound (gray shading indicates abundant common fragments that could not be annotated). The core peptide sequences for each compound are also shown. The structures of $\mathbf{2}$ and $\mathbf{3}$ were confirmed by NMR, while the structure of $\mathbf{4}$ is a proposal that correlates with $\mathrm{MS}^{2}$ data, the core peptide sequence, and predicted post-translational modifications.

270-272 $\mathrm{nm}$ (Figure S6), which is characteristic of thioamides. ${ }^{13}$ Notably, the $\mathrm{MS}^{2}$ data were consistent with $\mathrm{N}$ terminal lactyl (2) or pyruvyl (3 and 4) moieties, rather than the 2-hydroxy-2-methyl-4-oxopentanoyl group of $\mathbf{1}$.

Cloning and Heterologous Expression of the Thiostreptamide S4 Gene Cluster. To confirm that these molecules were indeed produced by thioviridamide-like pathways, we employed two different genetic approaches: gene disruption in a native producer strain and heterologous expression of a gene cluster. These approaches could both be achieved for the tsa cluster predicted to make 3 in Streptomyces sp. NRRL S-4. Gene cluster disruption was achieved by single crossover recombination between the $t s a H$ gene (encoding a $\mathrm{YcaO}$ domain protein ${ }^{14}$ ) and its truncated sequence cloned in pKC $1132^{15}$ to generate Streptomyces sp. NRRL S-4 $\Delta t s a H$. Production of 3 was abolished in this mutant strain (Figure 3), indicating that the gene cluster does indeed make this TLM. To support this result, we employed transformation associated recombination (TAR) cloning ${ }^{16-19}$ to generate a plasmid for heterologous expression of the tsa cluster. Here, a $19 \mathrm{kbp}$ sequence from Streptomyces sp. NRRL S-4 containing the putative thioviridamide-like gene cluster, as well as flanking regions up- and downstream, was cloned into the $\Phi$ C31

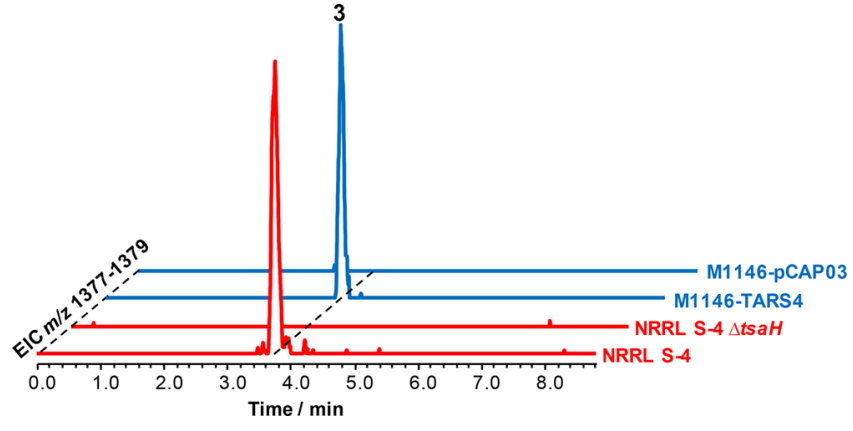

Figure 3. Production of thiostreptamide S4 in Streptomyces sp. NRRL S-4 and in S. coelicolor M1146-TARS4. Control strains unable to produce the compound are also shown.

integrative vector $\mathrm{pCAP03}^{20}$ using TAR in Saccharomyces cerevisiae VL6-48N. ${ }^{21}$ A successful clone (TARS4) was verified by PCR and restriction analysis and was introduced into Streptomyces coelicolor M1 $146^{22}$ by intergeneric conjugation. ${ }^{23}$ S. coelicolor M1146-TARS4 was cultured on solid BPM, and its LC-MS production profile was compared with a control strain containing an empty pCAP03 vector and wild type Streptomyces sp. NRRL S-4 as a positive control (Figure 3). S. coelicolor 
M1146-TARS4 produced a compound with $\mathrm{m} / z 1377.55$ that had an identical retention time and $\mathrm{MS}^{2}$ spectrum to those of 3 (Figure 3 and Figure S7), thereby proving that the cloned region is sufficient for thiostreptamide S4 biosynthesis.

Detailed Structural Analysis Reveals the Diversity within the Thioviridamide Family. To confirm the $\mathrm{MS}^{2}$ data and to pinpoint the location of additional post-translational modifications, fermentation cultures of the native producing strains were scaled up, and each compound was purified for structural elucidation by NMR. Compounds 2 and 3 were obtained in yields of 2 and $0.38 \mathrm{mg}$ per liter of solid culture from their respective native producers, and $1 \mathrm{D}$ and $2 \mathrm{D}$ NMR experiments $\left({ }^{1} \mathrm{H},{ }^{13} \mathrm{C}, \mathrm{COSY}, \mathrm{HSQC}, \mathrm{HMBC}\right.$, Figures S12-S24, Table S6) allowed us to establish their chemical structures. The ${ }^{13} \mathrm{C}$ NMR spectrum for thioalbamide (2, Figure 4) showed three downfield signals at $\delta_{\mathrm{C}} 206.7,207.0$, and 207.5

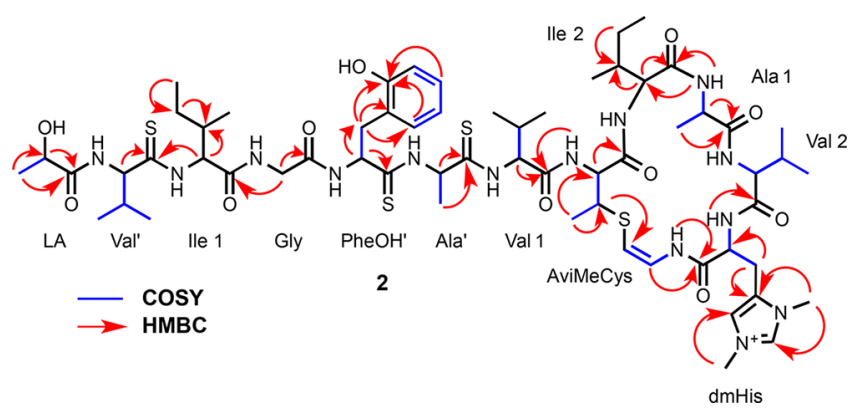

Figure 4. COSY and $\mathrm{HMBC}$ correlations identified for the structural characterization of 2 .

corresponding to nonprotonated carbons and indicating the presence of thioamide groups. These signals and their associated HMBC data correlate with the $\mathrm{MS}^{2}$ data for this compound, proving that the molecule has three thioamide linkages in the linear portion of the molecule. HMBC and COSY correlations also confirmed that the molecule has an AviMeCys-containing macrocycle, consistent with a Thr8 residue in the core peptide of TaaA instead of the Ser8 residue of the core peptide of 1. NMR analysis of the $N_{1}, N_{3^{-}}$ dimethylhistidinium residue showed that its $\beta$-carbon is a $\mathrm{CH}_{2}$ group $\left(\delta_{\mathrm{C}} 22.6\right)$ and therefore lacks the $\beta$-hydroxy group present in 1 . This is in agreement with the lack of a TvaJ-like 2oxoglutarate $/ \mathrm{Fe}$ (II)-dependent hydroxylase in the thioalbamide pathway.

$\mathrm{MS}^{2}$ data indicated that Phe5 is hydroxylated in 2, but the precise location of this modification could not be determined by $\mathrm{MS}^{2}$. The ${ }^{1} \mathrm{H}$ NMR spectrum of 2 showed the presence of four nonequivalent aromatic protons at $\delta_{\mathrm{H}} 6.83(1 \mathrm{H}$, ddd, 8.1, 8.0, 1.6), 6.87 (1H, dd, 8.0, 1.6), 7.12 (1H, ddd, 8.1, 8.0, 1.6), and $7.19(1 \mathrm{H}, \mathrm{dd}, 8.0,1.6)$, corresponding to protons on the 1 , 2,3 , and 4 positions of the phenyl group, and indicating that it is hydroxylated at the 5-position of the ring. Alongside the absence of a $\beta$-hydroxy group on histidine, this suggests that a cytochrome P450 (TaaCYP) encoded in the taa cluster does not functionally replace the hydroxylase absent from this pathway and instead catalyzes aromatic hydroxylation, thereby generating additional structural diversity within the TLM family. The predicted $N$-terminal lactate moiety of $\mathbf{2}$ was also confirmed based on the HMBC correlations between the methyl group at $\delta_{\mathrm{H}} 1.38(3 \mathrm{H}, \mathrm{d}, 6.8)$ with carbon signals at $\delta_{\mathrm{C}}$ 69.2 and 177.6. Interestingly, this is analogous to the $N$ terminus of JBIR-140, which is produced when the thioviridamide BGC is expressed in Streptomyces avermitilis SUKA17. ${ }^{24}$ We propose that a NAD $(\mathrm{P}) \mathrm{H}$-dependent reductase (TaaRED) catalyzes this reduction in the thioalbamide pathway, whereas it is likely that the reduction to generate JBIR-140 is catalyzed by a promiscuous reductase from $S$. avermitilis.

We were also able to pinpoint the post-translational modifications of 3 by analysis of the $\mathrm{MS}^{2}$ and NMR data. While it was not possible to establish full 2D NMR correlations throughout 3, four thioamide linkages were identified by HMBC data $\left(\delta_{\mathrm{C}} 200.6,201.2,202.2\right.$, and 204.6). HMBC correlations between a methyl group at $\delta_{\mathrm{H}} 2.35(3 \mathrm{H}, \mathrm{s})$ with carbon signals at $\delta_{\mathrm{C}} 160.4$ and 197.0, along with HMBC correlations between an amide proton at $\delta_{\mathrm{H}} 8.22$ with carbon signals at $\delta_{\mathrm{C}} 160.4$ and 63.1, allowed us to confirm the $N$ terminal pyruvyl group attached to $\mathrm{Val}^{\prime}$ (Figure S8). The $\mathrm{MS}^{2}$ fragment predicted to be the AviMeCys-containing macrocycle $(\mathrm{m} / z$ 687.33) matches a mass calculated from the core peptide and expected post-translational modifications if the macrocycle features a methyl group in addition to a hdmHis residue. Accordingly, the ${ }^{1} \mathrm{H}$ NMR spectrum displayed a singlet at $\delta_{\mathrm{H}}$ 3.71 and two equivalent aromatic protons at $\delta_{\mathrm{H}} 6.79(2 \mathrm{H}, \mathrm{d}$, $8.7)$ and $7.03(2 \mathrm{H}, \mathrm{d}, 8.7)$ that were consistent with $\mathrm{O}$ methylation of Tyr11, presumably catalyzed by the additional methyltransferase (TsaMT, pfam08242) encoded in the tsa gene cluster. The lack of any further oxidative modifications indicated that the flavin-dependent monooxygenase at the end of the gene cluster (TsaMO) is not involved in thiostreptamide S4 biosynthesis. Along with characteristic NMR signals, the presence of a hdmHis residue was supported by an $\mathrm{MS}^{2}$ fragment of $\mathrm{m} / z 125.07$ for 3 that concurs with retro-aldol fragmentation of the hdmHis residue (Figure S4) and was not present in the $\mathrm{MS}^{2}$ spectrum of 2 .

We were unable to obtain sufficient 4 for detailed NMR characterization, but its exact mass and $\mathrm{MS}^{2}$ spectrum (Figure S5) were fully consistent with the structure reported in Figure 2. This is in agreement with a lack of any additional tailoring enzymes encoded in the S-87 tsd gene cluster compared to the tva gene cluster (Figure 1c). $\mathrm{MS}^{2}$ data for 4 provided a macrocycle mass $(\mathrm{m} / z$ 657.31) that fits with the core peptide sequence assuming AviMeCys formation and the presence of the hdmHis residue. This is supported by $\mathrm{MS}^{2}$ fragmentation to generate $m / z$ 125.07, which was also seen for 3 and is indicative of the hdmHis residue. Notably, the y fragments of $\mathbf{4}$ signified an $\mathrm{N}$-terminal pyruvyl group. In some fermentation trials, compounds of $\mathrm{m} / z \quad 1393.5427$ and $\mathrm{m} / z \quad 1321.4813$ were produced by Streptomyces sp. NRRL S-4 and Streptomyces sp. NRRL S-87, respectively (Figures S9 and S10). These masses indicated the addition of one oxygen (calcd. $\mathrm{m} / z 1393.5416$ and $m / z \quad 1321.4841)$ to 3 and 4 , respectively. $\mathrm{MS}^{2}$ fragmentation of these molecule demonstrated that the sidechain sulfur on the Met3 residues on each compound are oxidized, shown by y fragments that are identical to their parent TLMs and the loss of methanesulfenic acid $\left(\mathrm{CH}_{3} \mathrm{SOH}, 64.00\right.$ $\mathrm{Da})$ from the parent ion, which is characteristic of oxidized methionine. ${ }^{25,26}$ The late onset and inconsistent production of these molecules indicates that they are generated by nonenzymatic oxidation during purification rather than being true pathway products.

A Nonenzymatic Origin of the Unusual $\mathrm{N}$-Terminus of Thioviridamide. One significant difference between the molecules reported here and $\mathbf{1}$ is at their $N$-termini, where $\mathbf{1}$ features a 2-hydroxy-2-methyl-4-oxopentanoyl group. Instead, 2 


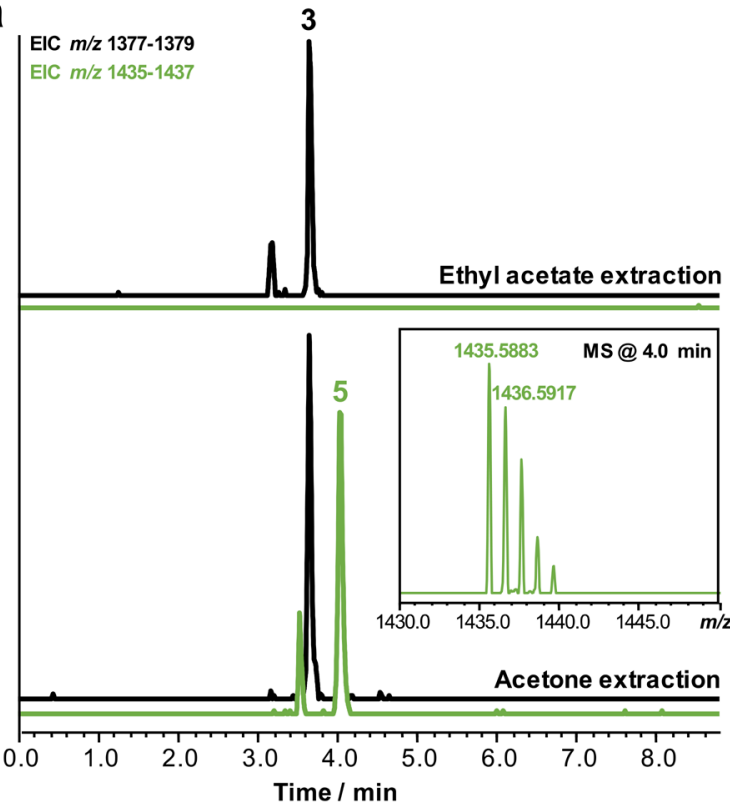

b

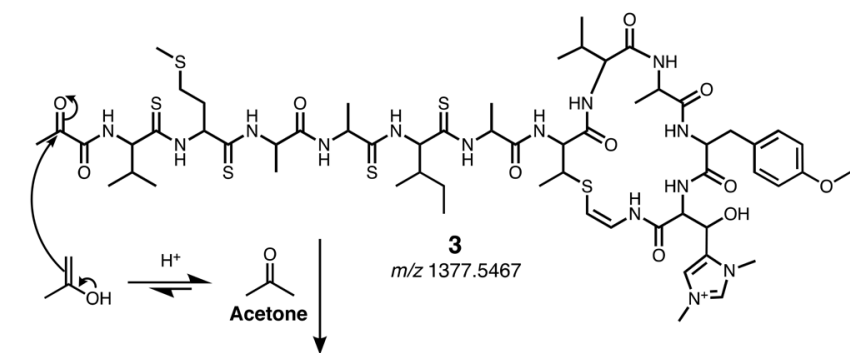

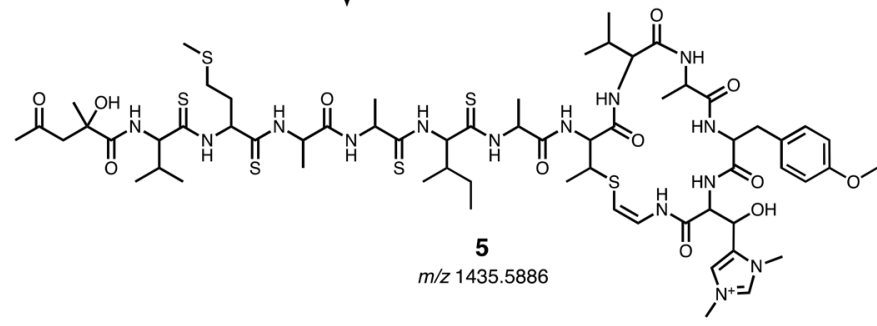

C

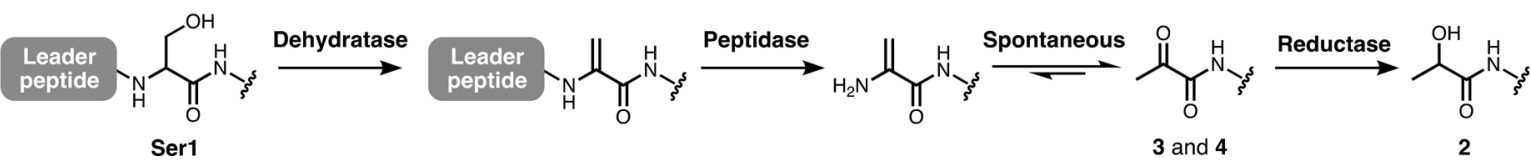

Figure 5. $\mathrm{N}$-terminal modification of TLMs. (a) LC-MS extracted ion chromatograms of S. coelicolor M1146-TARS4 extracted with either ethyl acetate (top) or acetone (bottom). (b) Generation of the $N$-terminal 2-hydroxy-2-methyl-4-oxopentanoyl group via reaction with acetone. (c) Biosynthetic proposal for the generation of the $\mathrm{N}$-terminal pyruvyl and lactyl moieties.

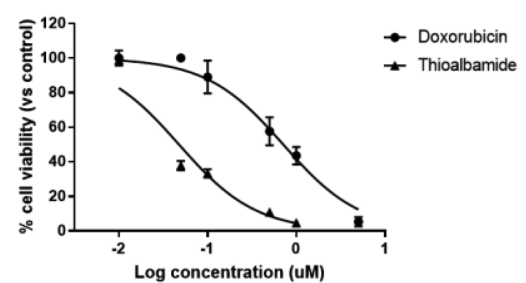

D

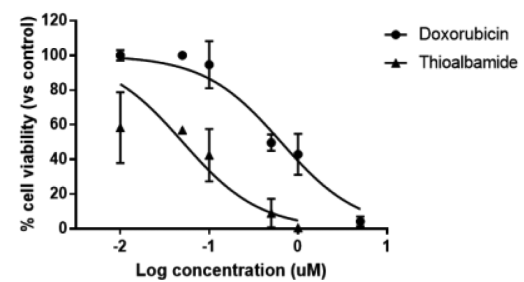

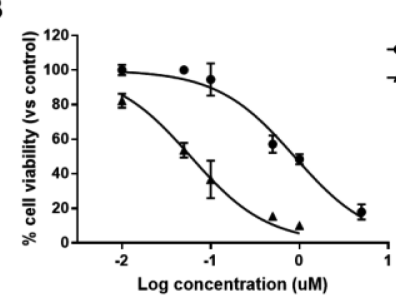

$\mathrm{E}$

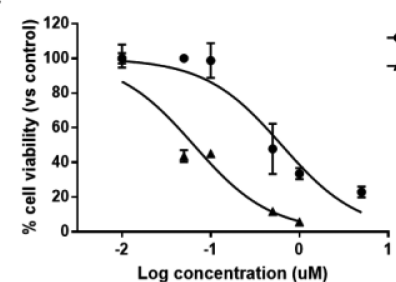

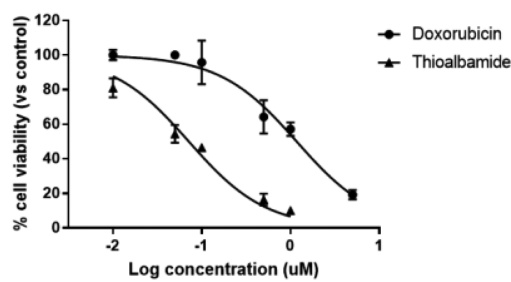

$\mathrm{F}$

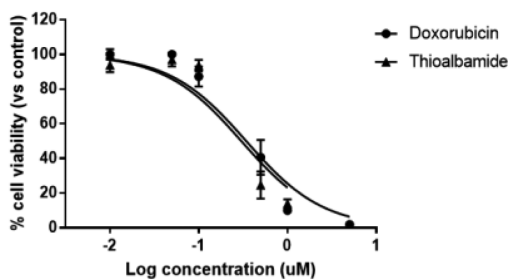

Figure 6. Effect of thioalbamide (2) on cell growth. Cellular growth assessment after treatment of A549 (A), MCF7 (B), MDA-MB-231 (C), HeLa (D), PA-TU-8988T (E), and MCF-10A (F) cell lines with different concentrations (0.1 to $1 \mu \mathrm{M})$ of 2 for $72 \mathrm{~h}$. Results, quantified by the MTT assay, are expressed as a percentage of growth versus control cells treated with DMSO. Values represent mean \pm SD of three independent experiments, each one performed with triplicate samples.

contains a lactyl moiety, while 3 and 4 each have pyruvyl groups. However, the tva gene cluster does not appear to encode any additional biosynthetic enzymes in comparison to the clusters reported here. This observation led us to speculate that the unusual $\mathrm{N}$-terminus of thioviridamide could feasibly be generated from an aldol reaction between a pyruvyl group and acetone, which was used in the published extraction of thioviridamide. ${ }^{5}$ In contrast, we used either methanol or ethyl acetate to extract our TLMs. We therefore used acetone to extract solid cultures of both Streptomyces sp. NRRL S-4 and S. coelicolor M1146-TARS4, which resulted in the production of a mixture of $3(\mathrm{~m} / z$ 1377.55) and a comparable amount of a compound with $m / z 1435.58$ (5, Figure 5a) that was not found when other solvents were used for extraction. $\mathrm{MS}^{2}$ data for $\mathbf{5}$ are consistent with an $\mathrm{N}$-terminal 2-hydroxy-2-methyl-4oxopentanoyl group (Figure S11), which implies that the true product of the thioviridamide pathway has an $\mathrm{N}$-terminal pyruvyl group (Figure $5 b$ ). Instead of being a post-translation- 
ally introduced modification, this pyruvyl group could derive from a dehydrated $\mathrm{N}$-terminal serine, which then spontaneously tautomerizes and exchanges with water following removal of the leader peptide (Figure 5c).

This result is consistent with a conserved serine residue at this position in almost all TLM precursor peptides (Figure 1b), and a homologous reaction has been proposed to be involved in the generation of the unusual $\mathrm{N}$-terminus of polytheonamide from threonine, ${ }^{27}$ the $\mathrm{N}$-terminal 2-oxobutanoyl group of lacticin $3147 \mathrm{~A} 2$, $^{28}$ and a pyruvyl group in a thiostrepton derivative generated by mutagenesis. ${ }^{29}$ The serine dehydration in TLM biosynthesis could feasibly be catalyzed by the same dehydratase that introduces the 2,3-didehydrobutyrine residue required for forming the AviMeCys macrocycle. Interestingly, the TLM gene clusters do not encode any Lan-like proteins that are usually required for dehydration, ${ }^{1}$ nor can a cypemycinlike mechanism occur, as this uses cysteine as a precursor to 2,3-didehydroalanine. ${ }^{30}$

Thioalbamide Is a Potent Anticancer Compound with Selective Activity. Thioviridamide has been reported to possess a potent antiproliferative effect against cancer cell lines. ${ }^{5}$ We were therefore interested in determining whether any of our newly discovered compounds exhibited comparable bioactivity. Compound $\mathbf{2}$ was therefore subjected to a series of activity assays on various model organisms, including both prokaryotic and eukaryotic systems. A wide panel of cancer cell lines were tested, including alveolar (A549), uterine cervical (HeLa), pancreatic (PA-TU-8988T), and luminal and basal breast (MCF7 and MDA-MB-231) adenocarcinoma cell lines (Figure 6). Compound 2 showed intense antiproliferative activity on all tumor lines tested with $\mathrm{IC}_{50}$ values ranging from 48 to $72 \mathrm{nM}$ (Table 1 ). Remarkably, the cytotoxic activity of 2

Table 1. Cytotoxic Activity of 2 in Comparison to Doxorubicin $^{a}$

\begin{tabular}{llll}
\multicolumn{1}{c}{ cell line } & & \multicolumn{1}{c}{ doxorubicin } & \multicolumn{2}{c}{$\mathbf{2}$} \\
A549 & IC $_{50}$ & 0.712 & 0.048 \\
& $95 \%$ confidence interval & 0.582 to 0.872 & 0.035 to 0.064 \\
MCF7 & IC $_{50}$ & 0.878 & 0.059 \\
& $95 \%$ confidence interval & 0.723 to 1.071 & 0.049 to 0.072 \\
MDA-MB-231 & IC $_{50}$ & 1.174 & 0.072 \\
& $95 \%$ confidence interval & 0.938 to 1,477 & 0.058 to 0.088 \\
HeLa & IC $_{50}$ & 0.644 & 0.050 \\
& $95 \%$ confidence interval & 0.487 to 0.852 & 0.027 to 0.090 \\
PA-TU-8988T & IC $_{50}$ & 0.630 & 0.065 \\
& $95 \%$ confidence interval & 0.451 to 0.888 & 0.047 to 0.089 \\
MCF 10A & IC $_{50}$ & 0.343 & 0.302 \\
& $95 \%$ confidence interval & 0.253 to 0.464 & 0.206 to 0.444
\end{tabular}

${ }^{a}$ Data are presented as $\mathrm{IC}_{50}$ values $(\mu \mathrm{M})$ and $95 \%$ confidence intervals obtained by nonlinear regression analysis of three independent experiments.

was found to be highly specific to tumor cells, as $\mathrm{IC}_{50}$ values on a nontumor breast epithelial cell line (MCF 10A) were 6 times higher than those found in cancer cells. This selectivity means that thioalbamide activity toward tumor lines is superior $(>10 \times$ lower $\mathrm{IC}_{50}$ ) to the clinically used doxorubicin but exhibits a comparable $\mathrm{IC}_{50}$ to doxorubicin toward the one healthy cell line we tested. In order to further investigate the specificity of the cytotoxic activity of 2 , it was tested against Gram positive (Staphylococcus aureus) and Gram negative (Escherichia coli, Klebsiella pneumoniae, and Pseudomonas aeruginosa) bacterial strains, and against the fungus Candida albicans. In each case, it did not inhibit the growth of the tested microorganisms, except for $S$. aureus, which was sensitive to high concentrations of 2, with a minimum inhibitory concentration (MIC) of $24 \mu \mathrm{M}$ (Table S7). At this concentration, however, activity was purely bacteriostatic and not bactericidal, as the minimum bactericidal concentration was over $48 \mu \mathrm{M}$.

\section{SUMMARY}

We report the genomics-guided discovery of three novel thioviridamide-like molecules and show that thioalbamide possesses nanomolar antiproliferative activity with approximately 6-fold selectivity for cancer cells over healthy cells. A recent analysis indicated that the TLMs are one of the rarest families of RiPP pathways across sequenced genomes, ${ }^{31}$ yet our work indicates that the family features a significant amount of structural diversity, both within the precursor peptide and by the array of modifying enzymes encoded in each cluster. TAR cloning was utilized to clone the thiostreptamide S4 (3) pathway, which was expressed in S. coelicolor M1146 to produce 3 at levels comparable to the wild type producing strain. This provides a promising platform for the future engineered production of novel TLMs, especially given the diversity identified across the 14 pathways that are currently sequenced. Our identification of these pathways should facilitate the characterization of the biosynthetic enzymes involved in the generation of these fascinating molecules. We propose that the reported $\mathrm{N}$-terminus of thioviridamide is an artifact of acetone extraction and that the true pathway product features an $N$ terminal pyruvyl group derived from serine. Finally, the newly identified TLM 2 possesses remarkable antiproliferative activity on all cancer cell lines that were tested, while being significantly less active toward a healthy nonmalignant cell line. Further work is required to determine whether $\mathbf{2}$ is similarly less active toward other noncancerous cell lines. The nanomolar levels of activity of $\mathbf{2}$ are superior to the clinically used anticancer agent doxorubicin across all tumor cell lines. This result indicates the power of genome mining to discover new anticancer molecules with clinical potential, and future studies will define the molecular mechanisms underlying the high pharmacological potential of the TLMs.

\section{ASSOCIATED CONTENT}

\section{S Supporting Information}

The Supporting Information is available free of charge on the ACS Publications website at DOI: 10.1021/acschembio.7b00677.

Experimental details, LC-MS and NMR spectra, and supporting figures and tables (PDF)

\section{AUTHOR INFORMATION}

\section{Corresponding Author}

*Tel.: +44(0)1603 450750. E-mail: andrew.truman@jic.ac.uk. ORCID

Andrew W. Truman: 0000-0001-5453-7485

Notes

The authors declare no competing financial interest.

\section{ACKNOWLEDGMENTS}

We would like to thank T. Eyles (John Innes Centre, U.K.) for assistance with TAR cloning, B. Wilkinson (JIC, U.K.) for 
helpful discussions and feedback on this manuscript, G. Saalbach (JIC, U.K.) for LC-MS assistance, and D. Howe and P. Grice (Department of Chemistry, University of Cambridge) for running NMR experiments. We are also very grateful to B. Moore (Scripps Institution of Oceanography, University of California San Diego, U.S.A.) for providing pCAP03, V. Larionov (National Cancer Institute, NIH, U.S.A.) for providing S. cerevisiae VL6-48N, and M. Bibb (JIC, U.K.) for providing S. coelicolor M1146. This work was supported by a Royal Society University Research Fellowship (A.W.T.) and the BBSRC MET ISP (BB/J004561/1) at the John Innes Centre (A.W.T. and R.L.).

\section{REFERENCES}

(1) Arnison, P. G., Bibb, M. J., Bierbaum, G., Bowers, A. A., Bugni, T. S., Bulaj, G., Camarero, J. A., Campopiano, D. J., Challis, G. L., Clardy, J., Cotter, P. D., Craik, D. J., Dawson, M., Dittmann, E., Donadio, S., Dorrestein, P. C., Entian, K.-D., Fischbach, M. A., Garavelli, J. S., Göransson, U., Gruber, C. W., Haft, D. H., Hemscheidt, T. K., Hertweck, C., Hill, C., Horswill, A. R., Jaspars, M., Kelly, W. L., Klinman, J. P., Kuipers, O. P., Link, A. J., Liu, W., Marahiel, M. A., Mitchell, D. A., Moll, G. N., Moore, B. S., Müller, R., Nair, S. K., Nes, I. F., Norris, G. E., Olivera, B. M., Onaka, H., Patchett, M. L., Piel, J., Reaney, M. J. T., Rebuffat, S., Ross, R. P., Sahl, H.-G., Schmidt, E. W., Selsted, M. E., Severinov, K., Shen, B., Sivonen, K., Smith, L., Stein, T., Süssmuth, R. D., Tagg, J. R., Tang, G.-L., Truman, A. W., Vederas, J. C., Walsh, C. T., Walton, J. D., Wenzel, S. C., Willey, J. M., and van der Donk, W. A. (2013) Ribosomally synthesized and post-translationally modified peptide natural products: overview and recommendations for a universal nomenclature. Nat. Prod. Rep. 30, 108-160.

(2) Truman, A. W. (2016) Cyclisation mechanisms in the biosynthesis of ribosomally synthesised and post-translationally modified peptides. Beilstein J. Org. Chem. 12, 1250-1268.

(3) Andes, D., Craig, W., Nielsen, L. A., and Kristensen, H. H. (2009) In vivo pharmacodynamic characterization of a novel plectasin antibiotic, NZ2114, in a murine infection model. Antimicrob. Agents Chemother. 53, 3003-3009.

(4) Dischinger, J., Basi Chipalu, S., and Bierbaum, G. (2014) Lantibiotics: promising candidates for future applications in health care. Int. J. Med. Microbiol. 304, 51-62.

(5) Hayakawa, Y., Sasaki, K., Adachi, H., Furihata, K., Nagai, K., and Shin-ya, K. (2006) Thioviridamide, a novel apoptosis inducer in transformed cells from Streptomyces olivoviridis. J. Antibiot. 59, 1-5.

(6) Sit, C. S., Yoganathan, S., and Vederas, J. C. (2011) Biosynthesis of Aminovinyl-Cysteine-Containing Peptides and Its Application in the Production of Potential Drug Candidates. Acc. Chem. Res. 44, 261268.

(7) Hayakawa, Y., Sasaki, K., Nagai, K., Shin-ya, K., and Furihata, K. (2006) Structure of thioviridamide, a novel apoptosis inducer from Streptomyces olivoviridis. J. Antibiot. 59, 6-10.

(8) De Zotti, M., Peggion, C., Biondi, B., Crisma, M., Formaggio, F., and Toniolo, C. (2016) Endothioxopeptides: A conformational overview. Biopolymers 106, 697-713.

(9) Izawa, M., Kawasaki, T., and Hayakawa, Y. (2013) Cloning and heterologous expression of the thioviridamide biosynthesis gene cluster from Streptomyces olivoviridis. Appl. Environ. Microbiol. 79, $7110-7113$.

(10) Nayak, D. D., Mahanta, N., Mitchell, D. A., and Metcalf, W. W. (2017) Post-translational thioamidation of methyl-coenzyme $\mathrm{M}$ reductase, a key enzyme in methanogenic and methanotrophic Archaea. eLife 6, e29218.

(11) Medema, M. H., Takano, E., and Breitling, R. (2013) Detecting sequence homology at the gene cluster level with MultiGeneBlast. Mol. Biol. Evol. 30, 1218-1223.

(12) Crone, W. J. K., Vior, N. M., Santos-Aberturas, J., Schmitz, L. G., Leeper, F. J., and Truman, A. W. (2016) Dissecting Bottromycin Biosynthesis Using Comparative Untargeted Metabolomics. Angew. Chem., Int. Ed. 55, 9639-9643.
(13) Huang, Y., Ferrie, J. J., Chen, X., Zhang, Y., Szantai-Kis, D. M., Chenoweth, D. M., and Petersson, E. J. (2016) Electronic interactions of $i, i+1$ dithioamides: increased fluorescence quenching and evidence for n-to- $\pi^{*}$ interactions. Chem. Commun. 52, 7798-7801.

(14) Burkhart, B. J., Schwalen, C. J., Mann, G., Naismith, J. H., and Mitchell, D. A. (2017) YcaO-Dependent Posttranslational Amide Activation: Biosynthesis, Structure, and Function. Chem. Rev. 117, 5389-5456.

(15) Bierman, M., Logan, R., O’Brien, K., Seno, E. T., Nagaraja Rao, R., and Schoner, B. E. (1992) Plasmid cloning vectors for the conjugal transfer of DNA from Escherichia coli to Streptomyces spp. Gene 116, $43-49$.

(16) Larionov, V., Kouprina, N., Graves, J., Chen, X. N., Korenberg, J. R., and Resnick, M. A. (1996) Specific cloning of human DNA as yeast artificial chromosomes by transformation-associated recombination. Proc. Natl. Acad. Sci. U. S. A. 93, 491-496.

(17) Kouprina, N., and Larionov, V. (2008) Selective isolation of genomic loci from complex genomes by transformation-associated recombination cloning in the yeast Saccharomyces cerevisiae. Nat. Protoc. 3, 371-377.

(18) Kim, J. H., Feng, Z., Bauer, J. D., Kallifidas, D., Calle, P. Y., and Brady, S. F. (2010) Cloning large natural product gene clusters from the environment: piecing environmental DNA gene clusters back together with TAR. Biopolymers 93, 833-844.

(19) Yamanaka, K., Reynolds, K. A., Kersten, R. D., Ryan, K. S., Gonzalez, D. J., Nizet, V., Dorrestein, P. C., and Moore, B. S. (2014) Direct cloning and refactoring of a silent lipopeptide biosynthetic gene cluster yields the antibiotic taromycin A. Proc. Natl. Acad. Sci. U. S. A. 111, 1957-1962.

(20) Tang, X., Li, J., Millán-Aguiñaga, N., Zhang, J. J., O’Neill, E. C., Ugalde, J. A., Jensen, P. R., Mantovani, S. M., and Moore, B. S. (2015) Identification of Thiotetronic Acid Antibiotic Biosynthetic Pathways by Target-directed Genome Mining. ACS Chem. Biol. 10, 2841-2849.

(21) Noskov, V., Kouprina, N., Leem, S.-H., Koriabine, M., Barrett, J. C., and Larionov, V. (2002) A genetic system for direct selection of gene-positive clones during recombinational cloning in yeast. Nucleic Acids Res. 30, 8e.

(22) Gomez-Escribano, J. P., and Bibb, M. J. (2011) Engineering Streptomyces coelicolor for heterologous expression of secondary metabolite gene clusters. Microb. Biotechnol. 4, 207-215.

(23) Kieser, T., Bibb, M. J., Buttner, M. J., Chater, K. F., and Hopwood, D. A. (2000) Practical Streptomyces Genetics, John Innes Foundation, Norwich.

(24) Izumikawa, M., Kozone, I., Hashimoto, J., Kagaya, N., Takagi, M., Koiwai, H., Komatsu, M., Fujie, M., Satoh, N., Ikeda, H., and Shinya, K. (2015) Novel thioviridamide derivative-JBIR-140: heterologous expression of the gene cluster for thioviridamide biosynthesis. J. Antibiot. 68, 533-536.

(25) Guan, Z., Yates, N. A., and Bakhtiar, R. (2003) Detection and characterization of methionine oxidation in peptides by collisioninduced dissociation and electron capture dissociation. J. Am. Soc. Mass Spectrom. 14, 605-613.

(26) Miles, C. O., Melanson, J. E., and Ballot, A. (2014) Sulfide oxidations for LC-MS analysis of methionine-containing microcystins in Dolichospermum flos-aquae NIVA-CYA 656. Environ. Sci. Technol. $48,13307-13315$.

(27) Freeman, M. F., Gurgui, C., Helf, M. J., Morinaka, B. I., Uria, A. R., Oldham, N. J., Sahl, H. G., Matsunaga, S., and Piel, J. (2012) Metagenome Mining Reveals Polytheonamides as Posttranslationally Modified Ribosomal Peptides. Science 338, 387-390.

(28) Cotter, P. D., Deegan, L. H., Lawton, E. M., Draper, L. A., O'Connor, P. M., Hill, C., and Ross, R. P. (2006) Complete alanine scanning of the two-component lantibiotic lacticin 3147: generating a blueprint for rational drug design. Mol. Microbiol. 62, 735-747.

(29) Li, C., Zhang, F., and Kelly, W. L. (2012) Mutagenesis of the thiostrepton precursor peptide at Thr7 impacts both biosynthesis and function. Chem. Commun. 48, 558-560.

(30) Claesen, J., and Bibb, M. (2010) Genome mining and genetic analysis of cypemycin biosynthesis reveal an unusual class of 
posttranslationally modified peptides. Proc. Natl. Acad. Sci. U. S. A. 107, 16297-16302.

(31) Skinnider, M. A., Johnston, C. W., Edgar, R. E., Dejong, C. A., Merwin, N. J., Rees, P. N., and Magarvey, N. A. (2016) Genomic charting of ribosomally synthesized natural product chemical space facilitates targeted mining. Proc. Natl. Acad. Sci. U. S. A. 113, E6343E6351. 